\title{
Thermoluminescence energy response of a germanium-doped optical fiber obtained using a Monte Carlo N-particle code simulation
}

\begin{abstract}
This paper reports on the energy response of a Ge-doped optical fiber subjected to photon irradiation. The thermoluminescence (TL) responses of the Ge-doped optical fiber for various photon energies ranging from $20 \mathrm{keV}$ to $6 \mathrm{MeV}$ were investigated as energy absorbed in the TL material by using the Monte Carlo N Particle transport code version 5 (MCNP5). The results obtained are compared against results for the thermoluminescence dosimeter 100 (TLD-100). The input parameters included in this study were the geometry specification, the source information, the material information and tallies. Tally F6 is an important parameter in data card which was used in this simulation as a energy-dependant heating function instead of flux. Similar patterns of response were found for each dosimeter. The simulation shows that the optical fiber has a greater response than TLD 100 in the lower energy range, but the responses overlap in the higher energy ranges.
\end{abstract}

Keyword: Thermoluminescence; Energy response; Ge-doped optical fiber; TLD 100; Photon; MCNP5 